\title{
Apport des lasers en odontologie
}

\section{Benefit of laser in odontology}

\section{MOTS-CLEFS :}

- Laser, longueur d'onde, énergie, puissance,

fluence, absorption

\section{KEYWORDS:}

- Laser, wave length, energy, power, fluence, absorption

\section{Résumé}

Depuis une quinzaine d'années, le laser est utilisé avec succès en dentisterie. Chaque laser est spécifique par sa longueur d'onde. L'absorption du rayonnement laser dans les tissus durs ou mous dépend de cette longueur d'onde.

L'expérimentation et la pratique du laser dans les universités permettra de mieux traiter les patients en garantissant le bon usage et le choix de la longueur d'onde adaptée.

\section{Abstract}

Since about fifteen years, Lasers has been successfully used in dentistry. Each laser is specific by his wave length. The absorption of the light beam in hard of soft tissues depends of this wave length.

The laser's practice in the Universities offers the best treatment for the patients by the good procedure and the good choice of the wave length.
AOS 2015;272:2-14 DOI: $10.1051 / \mathrm{aos} / 2015202$ (C) EDP Sciences 2015

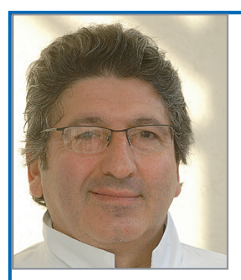

- Jean-Michel STROUMZA, Docteur en Chirurgie Dentaire, Paris 5 CES de diététique et nutrition humaine, CES de Prothèse Fixée, Paris 7 DU d'Implantologie Chirurgie et Prothèse, Paris 7 - DU Chirurgie Pré et Péri-Implantaire de Paris 11 - DU Européen en Chirurgie dentaire Laser Assistée Certificat d'Hypnose Médicale - Ex AHU en Chirurgie Buccale, Paris 7 Attaché de Consultation Service Implantologie, Paris 7

www.laserimplantdentaire.com - stroumza.jm@gmail.com 110 rue La Boétie 75008 Paris 


\section{INTRODUCTION}

Le laser a été mis en "lumière " par A. Einstein en 1917, lorsqu'il publia ses travaux sur les notions d'absorption de lumière par la matière, puis par son émission spontanée et stimulée. Il posa ainsi les bases scientifiques du LASER dont l'acronyme signifie : Light Amplification by Stimulated Emission of Radiation.

Il aura fallu attendre près d'un demi-siècle pour que ses applications thérapeutiques voient le jour, grâce à Maiman qui, en 1960, réalisa le premier oscillateur à rubis, ouvrant ainsi la voie de l'utilisation du laser en médecine. La même année, le premier laser à gaz avec un mélange hélium et néon est mis au point. Puis, en 1962, les premiers lasers diode sont créés et, en 1964, apparaissent des lasers à gaz $\mathrm{CO}_{2}$.

De nombreux lasers vont voir le jour. En 1970, alors que Kaminsky découvre le pérovskite qui rentre dans la composition du laser Nd-YAP (yttrium, aluminium et pérovskite), on observe une utilisation croissante des lasers en médecine, en ophtalmologie, en ORL, en chirurgie, etc. En 1973, le laser Nd-YAG est mis au point. En 1980, naissance des lasers Erbium-YAG. En 1994, mise au point du Nd-YAP.

Quarante-trois ans ont séparé la théorie de la pratique, trente ans ont séparé le début de mon cursus odontologique et ma première utilisation du laser en thérapeutique dentaire.

Les lasers utilisés en pratique quotidienne sont choisis pour leurs effets spécifiques, leurs interactions tissulaires sous la dépendance de leur longueur d’onde.

Tout laser se définit par sa longueur d'onde. On distingue :

$\checkmark$ les diodes;

$\checkmark$ le laser $\mathrm{CO}_{2}$;

$\checkmark$ le laser Nd-YAG;

$\checkmark$ le laser Nd-YAP ;

Ile laser Erbium-YAG.

Les lasers Nd-YAP et Erbium-YAG prouveront leur efficacité à la lumière de trois cas cliniques :

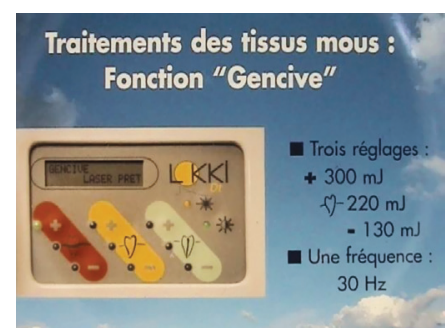

$\triangle$ Fig. 1 : Laser Nd-YAP.

Fig. 2 : Trois fréquences et trois réglages par fréquence.

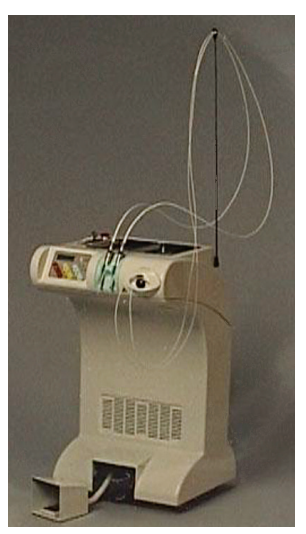

parodontologie : traitement de poches parodontales sur piliers de bridge maxillaire;

$\checkmark$ élongation coronaire sur une molaire mandibulaire très égressée ;

I endo-implanto : traitement d'une sinusite maxillaire dorigine endodontique et préparation implantaire en vue d'une réhabilitation occluso-prothétique maxillaire totale.

\section{CHOIX DU MATÉRIEL}

En pratique quotidienne, on utilise deux lasers dont les indications sont complémentaires : le Nd-YAP et l'Erbium-YAG.

\section{Nd-YAP}

Le laser Nd-YAP vit le jour en 1994. C'est un laser solide qui utilise un cristal d'yttrium aluminium pérovskite dopé au néodyme (fig. 1).

Sa longueur d'onde de $1340 \mathrm{~nm}$ fait que ce laser est bien absorbé dans l'eau et le sang. Sa pénétration est alors de 150 microns dans les tissus, donc plus absorbé que le Nd-YAG et la diode [38] dont la pénétration du rayonnement avoisine les $10 \mathrm{~mm}$ et moins absorbé que le $\mathrm{CO}_{2}$ (10 à 20 microns) ou l'Erbium-YAG (2 à 5 microns).

Lémission du rayonnement laser se fait par des impulsions très courtes, de l'ordre de 150 microsecondes, entrecoupées de périodes de repos. La fréquence de l'impulsion varie de 5, 10 ou 30 hertz (fig. 2).

Il est réglable sur trois fréquences (fig. 2) avec trois réglages par fréquence et muni de deux fibres (fig. 3) de $200 \mu$ permettant le traitement endodontique des canaux très fins et $320 \mu$ pour la parodontologie et la chirurgie, avec un aiguilleur de fibres permettant un changement immédiat (fig. 4).

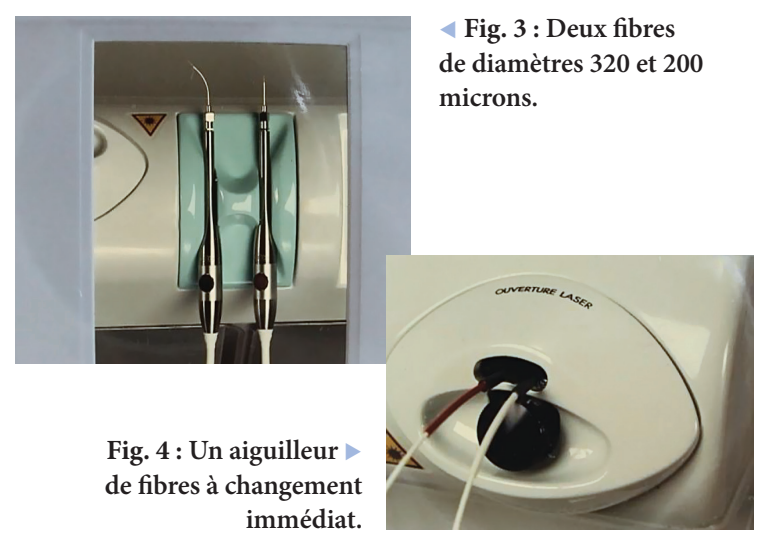




\section{Erbium-YAG}

Dans le laser Er-YAG, le cristal d'yttrium aluminium garnet est dopé à l'erbium. La longueur d'onde de son rayonnement de $2940 \mathrm{~nm}$ se trouve à un pic maximum d'absorption de l'eau et de l'hydroxyapatite. Il est donc très efficace sur les tissus durs. Lémission du rayonnement laser se fait par des impulsions très courtes de lordre de 240 microsecondes entrecoupées de périodes de repos.

La fréquence de l'impulsion varie de 4 à 50 hertz (fig. 5).

L'énergie de chaque impulsion (exprimée en millijoules) peut être réglée par l'opérateur. La combinaison de la fréquence et de l'énergie des impulsions permet de calculer la puissance moyenne mesurée en watts (W). Le système de transmission se fait soit par deux fibres de diamètre 200 et 300 microns pour le YAP (fig. 3 et 4), soit par des fibres optiques à base de saphir ou de quartz pour l'Er-YAG (fig. 6).

Le principe actif de l'Er-YAG est l'ablation des tissus mous, des tissus osseux ou dentaires par le principe de la vaporisation explosive due à l'absorption de l'énergie par les molécules d'eau qui, en sévaporant, provoquent des micro-explosions superficielles à l'origine de la vaporisation des tissus.

Le rayonnement laser pulsé de ces deux lasers a une puissance de crête importante (jusqu'à $2,6 \mathrm{~kW}$ ).

Ces deux lasers seront étudiés au travers de plusieurs cas cliniques.

Le laser est déterminé par sa longueur d’onde. Chaque longueur d'onde possède une absorption spécifique dans les différents constituants des tissus biologiques : eau, hémoglobine, hydroxyapatite et mélanine (fig. 7).

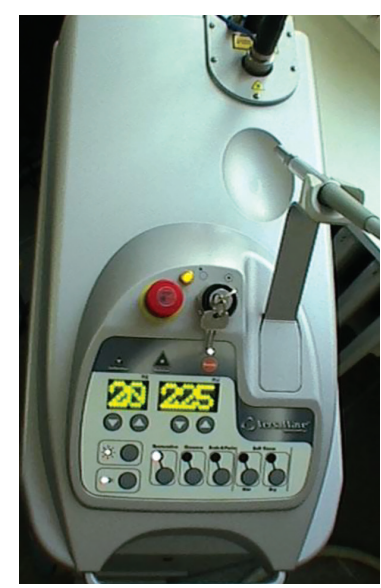

$\triangleleft$ Fig. 5 : Laser Erbium-YAG.

$\checkmark$ Fig. 6 : Vue latérale.

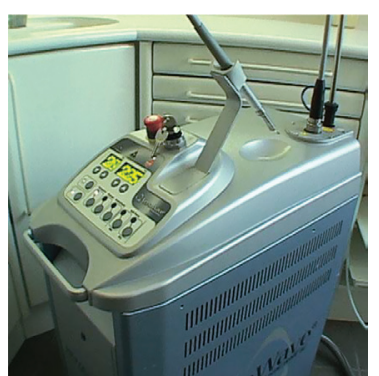

Ce tableau montre que le laser Erbium-YAG est le plus fortement absorbé dans l'eau donc son pouvoir de pénétration tissulaire sera faible (entre 2 et 15 microns). Le Nd-YAP, moins absorbé que l'erbium dans l'eau mais beaucoup plus que la diode ou le laser Nd-YAG (environ 20 à 30 fois plus), aura un pouvoir de pénétration tissulaire de 150 microns par rapport aux deux autres qui peuvent atteindre 2 à 3000 microns. Ces éléments doivent être connus et intégrés pour ne pas nuire aux tissus rencontrés et être vigilant et prudent lors de l'utilisation du laser.

En revanche, les lasers Nd-YAP et Nd-YAG seront plus hémostatiques que l'Erbium-YAG.

Le schéma (fig. 8) illustre bien les interactions spécifiques entre différents lasers et différents tissus. $\mathrm{CO}_{2}$ et erbium agiront sur la cornée uniquement alors que le laser Nd-YAG ou diode traverseront la cornée et d'autres structures sans rien modifier pour agir plus loin sur la rétine [9].

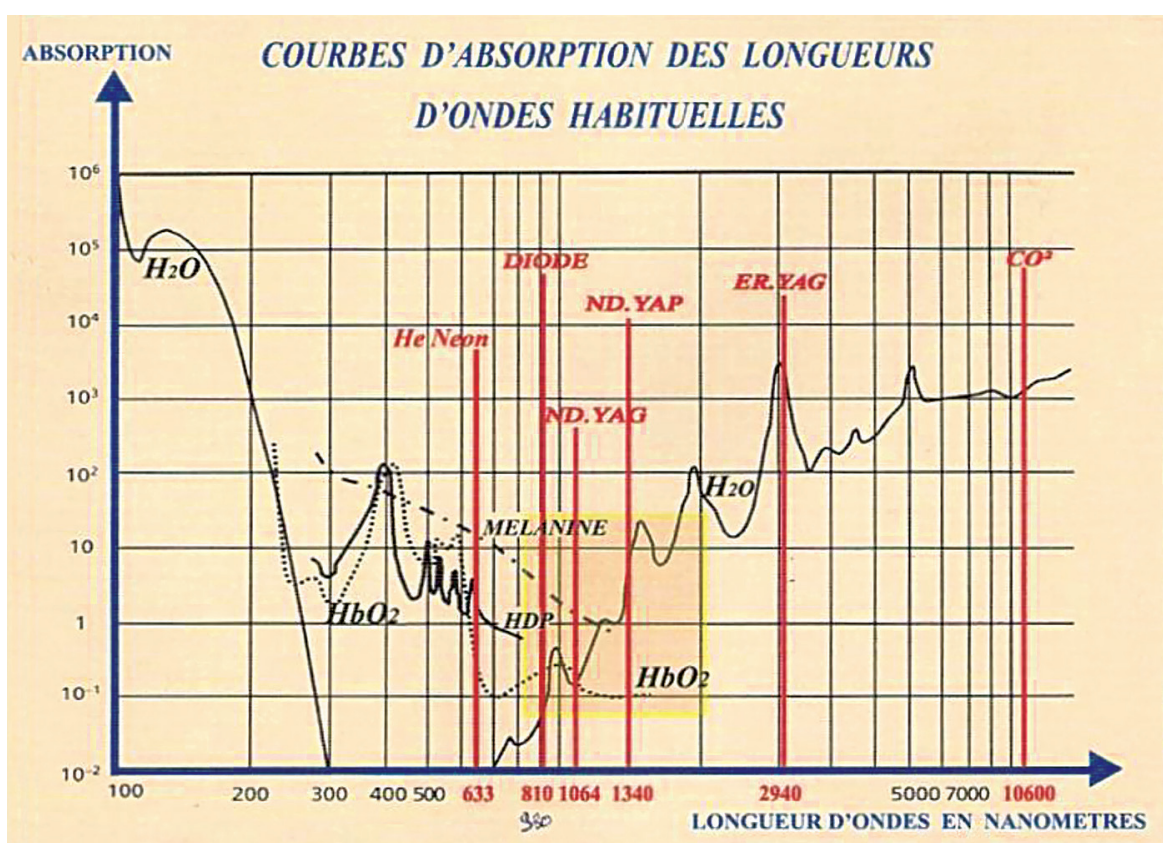

4 Fig. 7 : Diagramme

d'absorption des lasers dans les différents constituants des tissus biologiques [18]. 
Le schéma (fig. 9) montre que le rayonnement laser qui réfléchit ou traverse un tissu ne modifiera pas ce dernier contrairement à l'absorption ou la diffusion qui transformeront les tissus sur lesquels le laser agira [9].

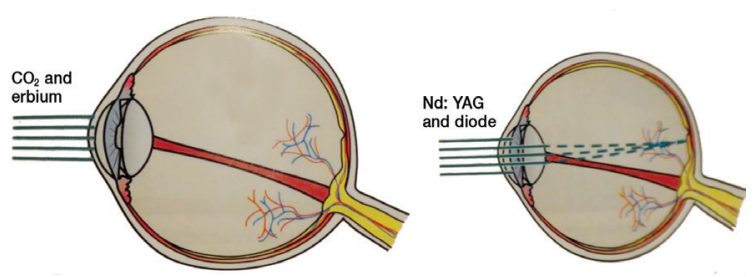

$\triangle$ Fig. $8: \mathrm{CO}_{2}$ et erbium agissent sur la cornée, diode et Nd-YAG sur la rétine [9].

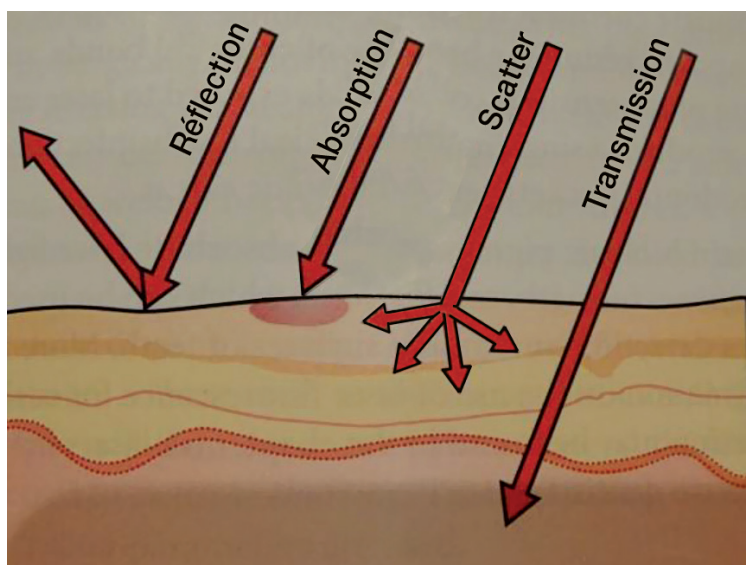

$\triangle$ Fig. 9: Quatre interactions possibles entre lasers et tissus [9].

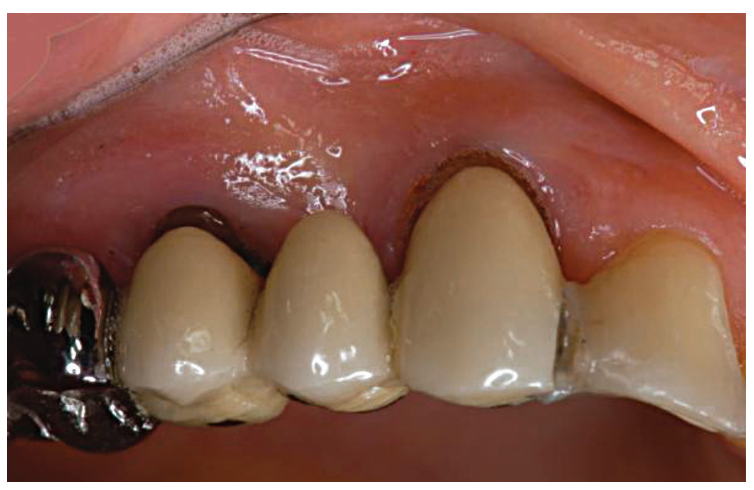

\section{CAS CLINIQUES}

Nous verrons successivement un cas de parodontite avancée, une élongation coronaire, une sinusite d'origine dentaire et une péri-implantite :

parodontologie : poches parodontales profondes sur pilier de bridge maxillaire ;

$\checkmark$ élongation coronaire : traitement complet d'une molaire mandibulaire;

I sinusite iatrogène : traitement d'une sinusite maxillaire d'origine endodontique et préparation implantaire en vue d'une réhabilitation occluso-prothétique maxillaire totale ;

$\checkmark$ péri-implantite : traitement d'une péri-implantite subterminale au maxillaire.

\section{Parodontite avancée}

\section{Phase initiale}

Le bridge maxillaire droit de cette patiente est très mobile (fig. 10). Son praticien lui propose l'extraction des trois piliers ancrage de bridge très alvéolysés et son remplacement par une prothèse adjointe amovible de cinq éléments. Ne souhaitant pas de prothèse amovible, elle demande un avis complémentaire à un autre praticien.

Après avoir été informée de l'utilisation du laser dans son cas, plan de traitement, devis et consentement éclairé sont établis.

\section{Phase préopératoire}

Sous anesthésie, la matérialisation des poches parodontales se fait en introduisant des cônes de gutta dans toutes les poches. La radio rétroalvéolaire (fig. 11) fixe la limite apicale de la poche (fig. 12). La sonde parodontale mesure la profondeur des poches et les mesures sont notées sur la fiche du patient (fig. 13).

Le bridge mobile est alors solidarisé par du composite au bloc antérieur plus stable. Maxillaire et mandibule sont détartrés. Le secteur maxillaire droit est alors bétadiné.

$\triangle$ Fig. 10 : État initial : bridge de 4 éléments très mobiles.

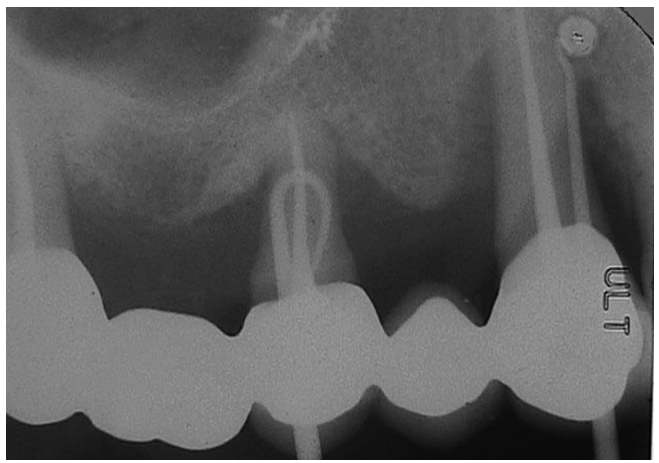

$\triangle$ Fig. 11 : Radio et mise en évidence des poches parodontales profondes.

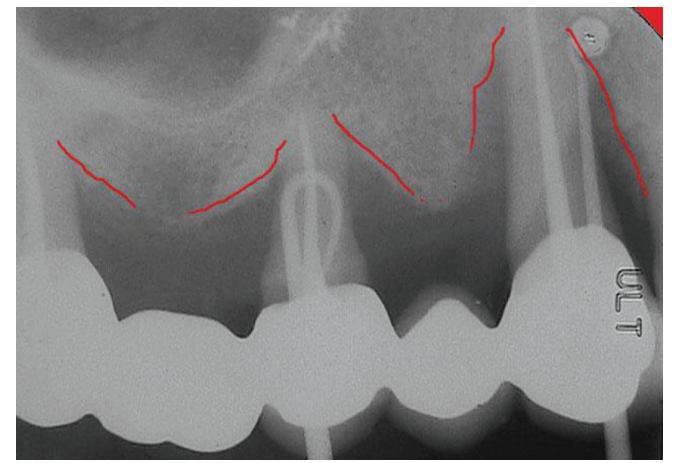

$\triangle$ Fig. 12 : Matérialisation des PP. 


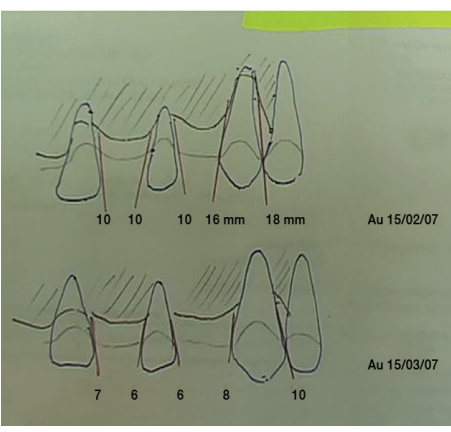

$\triangle$ Fig. 13 : Schéma de lévolution en 1 mois.

\section{Phase du traitement laser}

Les poches sont irriguées avec une solution de peroxyde d'hydrogène à 10 volumes, sans aucune pression, laissée in situ pendant quelques secondes. Le laser est réglé sur G- $(30 \mathrm{~Hz}-130 \mathrm{~mJ})$ et après validation, la fibre laser du Nd-YAP est introduite au fond de la poche dans un bain $\mathrm{d}^{\prime} \mathrm{H}_{2} \mathrm{O}_{2}$. L'appui sur la pédale déclenche alors des suites de tirs lasers en rafale. La fibre de 300 microns doit impérativement être en mouvement dans la poche bien irriguée. Les tirs sont entrecoupés de phases de repos pour laisser aux tissus le temps de récupérer et éviter ainsi la surchauffe néfaste à la régénération des tissus [1]. Le diamètre de la fibre fait 320 microns et la position de la puissance est G- ; c'est-à-dire 30 impulsions par seconde de 150 microsecondes à la puissance moyenne de 5 watts.

\section{Phase chirurgicale}

Des curettes très fines sont parfois utilisées pour éliminer le tissu de granulation non volatilisé par l'action laser et qui par déshydratation réduit le résidu de poche facilement éliminé à la sonde.

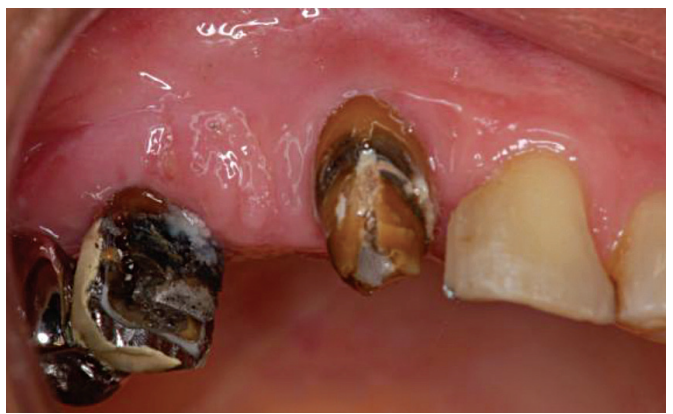

$\triangle$ Fig. 16 : Situation à la dépose du bridge.

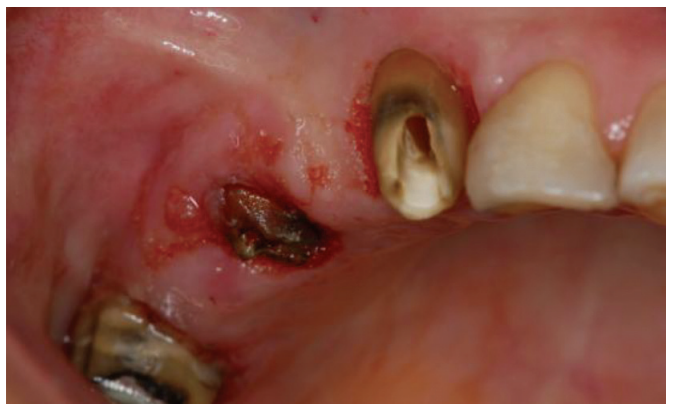

$\triangle$ Fig. 18 : État assaini après action laser.

\section{Phase post-chirugicale}

Un mois plus tard, le sondage de poches montre une diminution significative des profondeurs mesurées à la première séance (fig. 13 et 14).

\section{Phase pré-prothétique post-chirugicale}

A ce stade de consolidation, un projet est proposé aux patients (fig. 15).

\section{Phase prothétique}

Lancien bridge est déposé sept mois plus tard (fig. 16); il est remplacé par un bridge provisoire qui fera office de guide radiologique puis chirurgical après avoir traité les dents préparées et la gencive au laser sous irrigation abondante d' $\mathrm{H}_{2} \mathrm{O}_{2}$ (fig. 17 et 18). À ce stade, les piliers stabilisés vont recevoir des inlay-cores (fig. 19) car la dépose du bridge aux limites cervicales peu étanches met en évidence un délabrement coronaire avancé.

Le laser va durcir le tissu dentinaire et assainir la gencive périphérique [15].

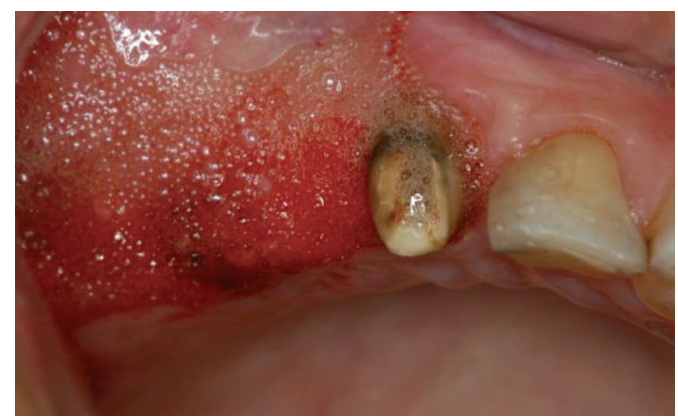

$\triangle$ Fig. 17 : Irrigation $\mathrm{H}_{2} \mathrm{O}_{2} 10$ volumes.

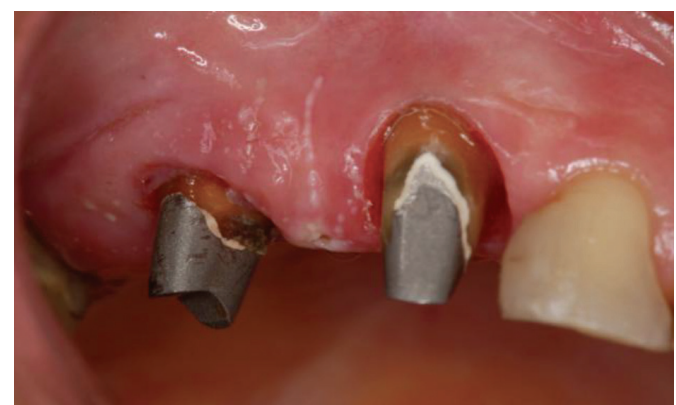

$\triangle$ Fig. 19 : Scellement des inlay-cores. 


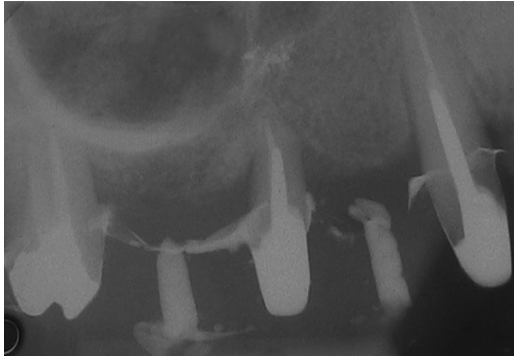

$\triangle$ Fig. 20 : Radio guide radiologique en place.

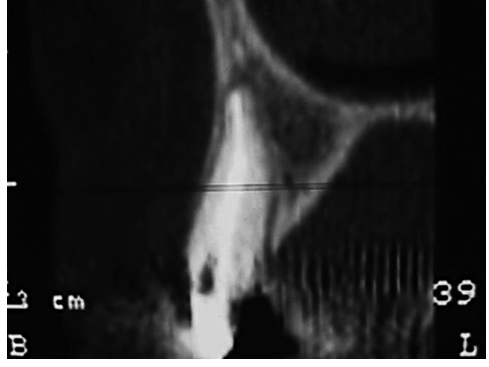

$\triangle$ Fig. 21 : Coupe coronale en 13.

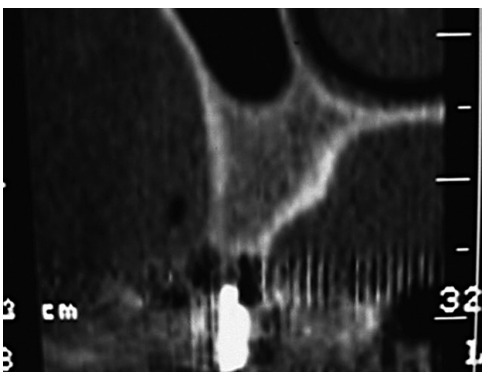

$\triangle$ Fig. 22 : Coupe coronale en 14.

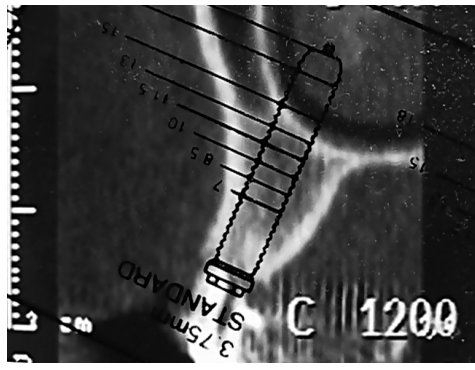

$\triangle$ Fig. 23 : Projet implantaire avec transparent.

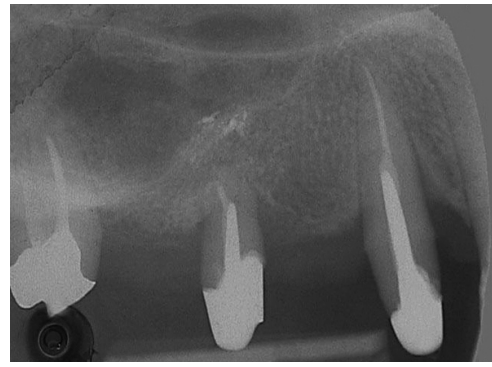

$\triangle$ Fig. 24 : État stable préimplantaire.

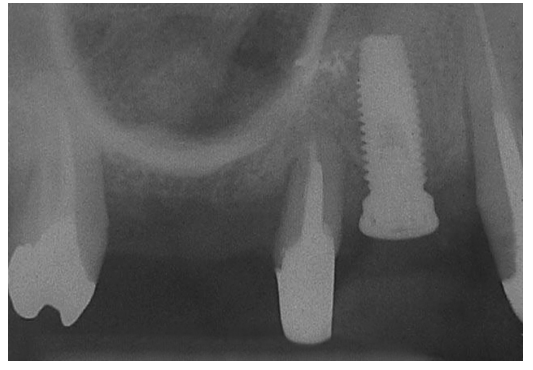

$\triangle$ Fig. 25 : Situation postimplantaire.

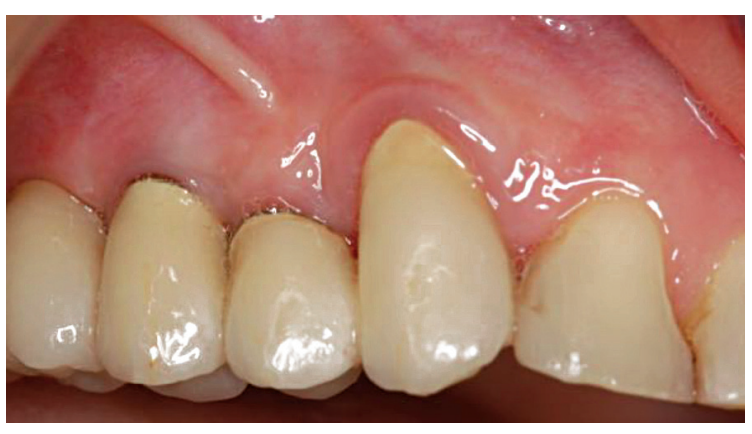

$\triangle$ Fig. 26 : Bridge définitif.

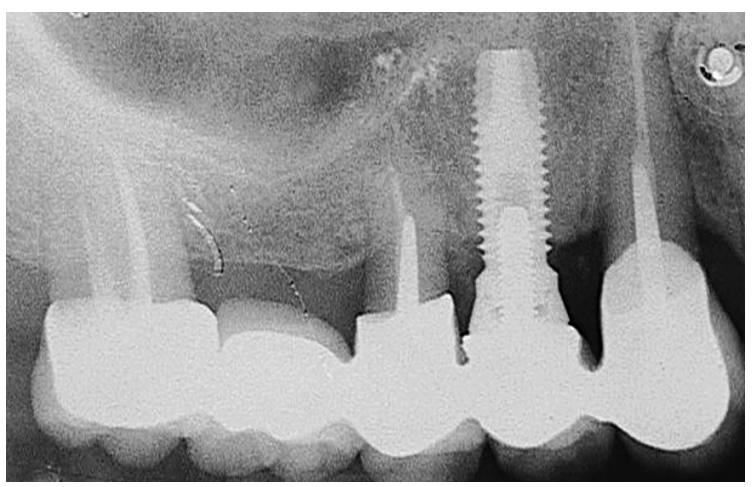

$\triangle$ Fig. 27 : Radio de contrôle.

Fig. 28 : Radio initiale.

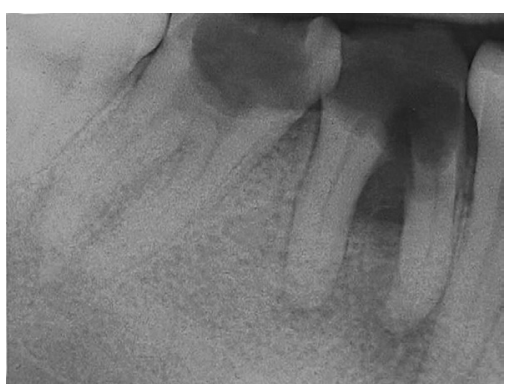

\section{Suivi}

À huit mois : réalisation d'un scanner objectivant la reconstruction du tissu osseux (fig. 20, 21, 22 et 23) et pose d'un implant (fig. 24 et 25) $[2,3]$.

À douze mois : un bridge implanto-dento-porté est scellé (fig. 26) et un contrôle radiologique met en évidence la reconstruction ad integrum du tissu osseux (fig. 27).

\section{Conclusions}

Lénergie laser montre son pouvoir régénérateur et permet l'accélération de la cicatrisation des tissus traversés. Un facteur reste cependant fondamental : la participation active du patient. Son implication et son observance sont proportionnelles au résultat ; c'est la clé du succès en laserothérapie.

\section{Élongation coronaire}

Le traitement laser est réalisé sur une patiente de 35 ans pour rétablir une occlusion équilibrée et faciliter la reconstruction prothétique d'une molaire très égressée, très délabrée par la carie (fig. 28 et 29) et le manque

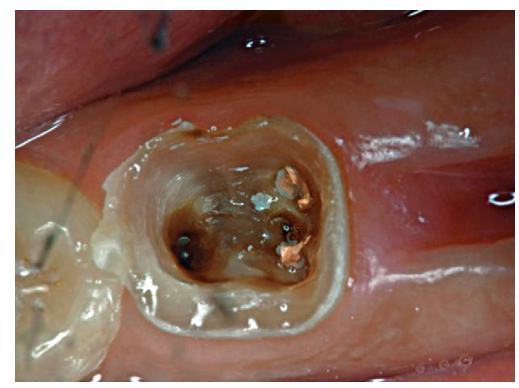

$\triangle$ Fig. 29: Situation clinique après extraction de 46. 


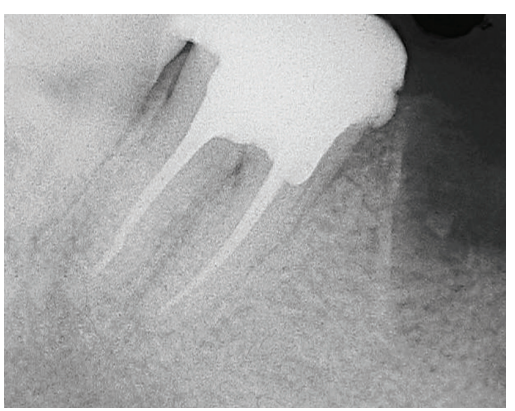

$\triangle$ Fig. 30 : Traitement endo-laser assisté de la 47.

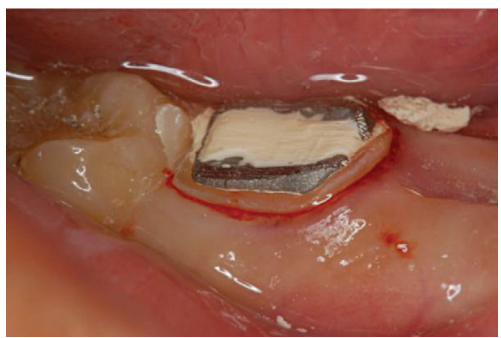

$\triangle$ Fig. 33 : Scellement du faux moignon.

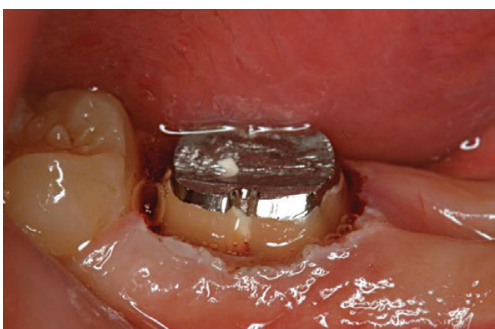

$\triangle$ Fig. 36 : Après intervention.

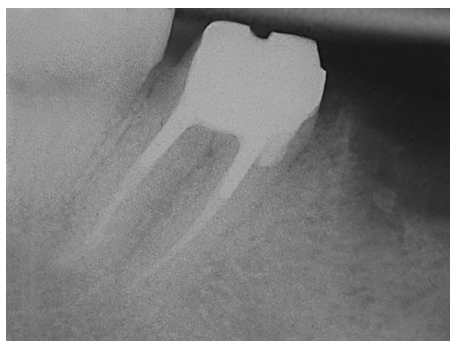

$\triangle$ Fig. 39 : Radio de contrôle.

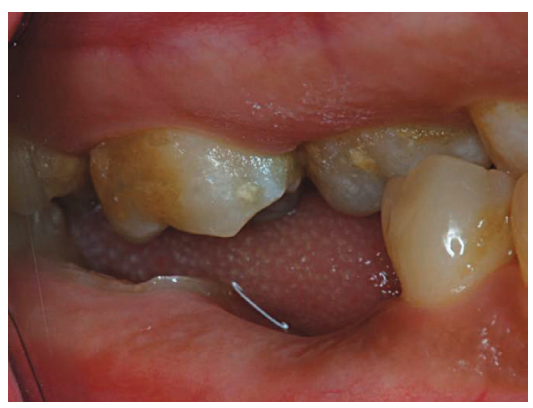

$\triangle$ Fig. 31 : Situation interarcade défavorable 14.

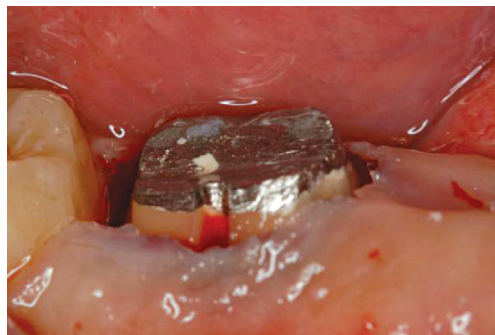

$\triangle$ Fig. 34 : Élongation coronaire laser assistée.

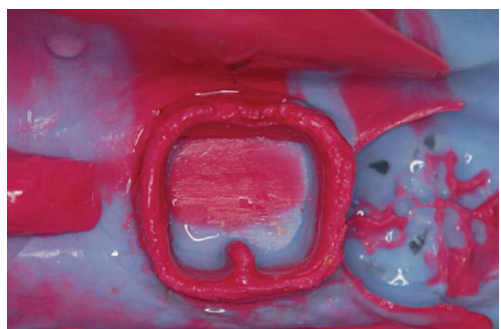

$\triangle$ Fig. 37 : Prise d'empreinte.

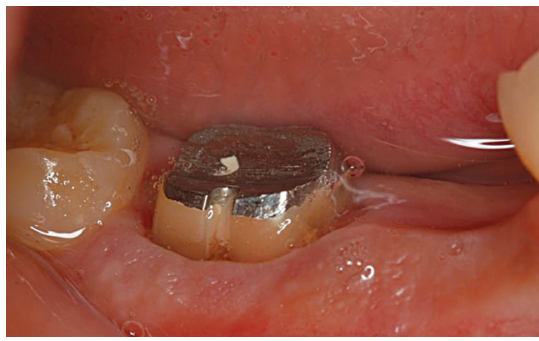

$\triangle$ Fig. 40 : État gingival à 7 jours.

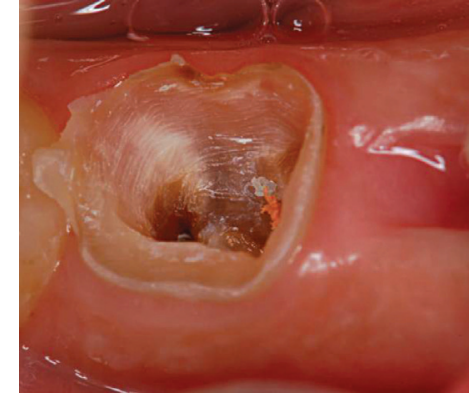

$\triangle$ Fig. 32 : Préparation avant empreinte de l'inlay-core (traitement dentinaire laser assisté).

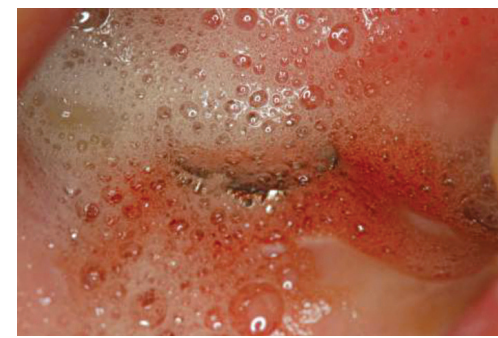

$\triangle$ Fig. 35 : Association $\mathrm{H}_{2} \mathrm{O}_{2} 10$ volumes.

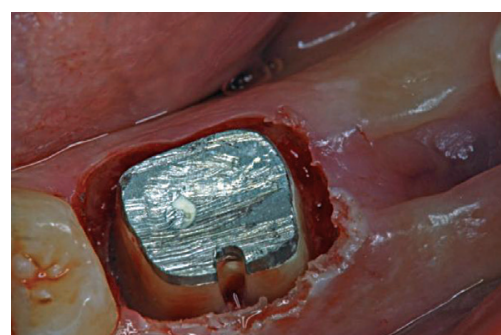

$\triangle$ Fig. 38 : Situation en fin de séance.

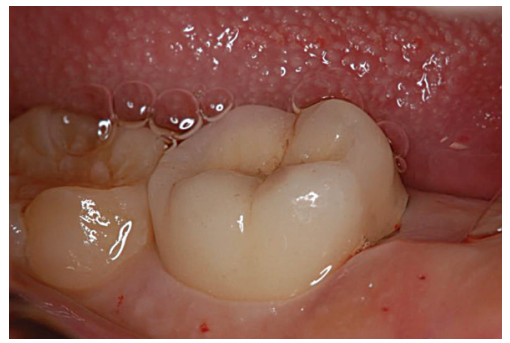

$\triangle$ Fig. 41 : Pose de la CCM. de soins. Après extraction de $46[2,25]$ et traitement endodontique de la dent 47 (fig. 30) [8], réalisation d'une élongation coronaire afin de permettre de couronner cette dent dans de bonnes conditions (espace biologique supra-osseux et espace interarcade favorable pour assurer la pérennité de cette dent).

Dans ce cas, le laser est une aide précieuse dans la décontamination dentinaire et l'assurance d'avoir une obturation radiculaire de qualité. Le peu d'espace interarcade lors de la pose du faux moignon juxtagingival (fig. 31, 32 et 33) oblige à réaliser une élongation coronaire (fig. 34). La gingivorragie provoquée par la fraise dans l'os alvéolaire et la gencive marginale est alors jugulée par l'action hémostatique du laser Nd-YAP dont la fibre de 320 travaille par quart de tour sous une bonne irrigation délivrée par la seringue à eau oxygénée 10 volumes (fig. 35). Rappelons que la température sélève à $1500^{\circ} \mathrm{C}$ à sec et diminue à $700^{\circ} \mathrm{C}$ sous irrigation.

Une minute plus tard, l'empreinte peut être prise dans d'excellentes conditions (fig. 36 et 37 ). Après retrait de l'empreinte, on peut lire la limite cervicale de la dent préparée totalement exsangue (fig. 38), contrôlée radiologiquement (fig. 39). Sept jours plus tard, la dent 47 entourée d'un manchon de gencive attachée de belle texture (fig. 40) est couronnée (fig. 41). 


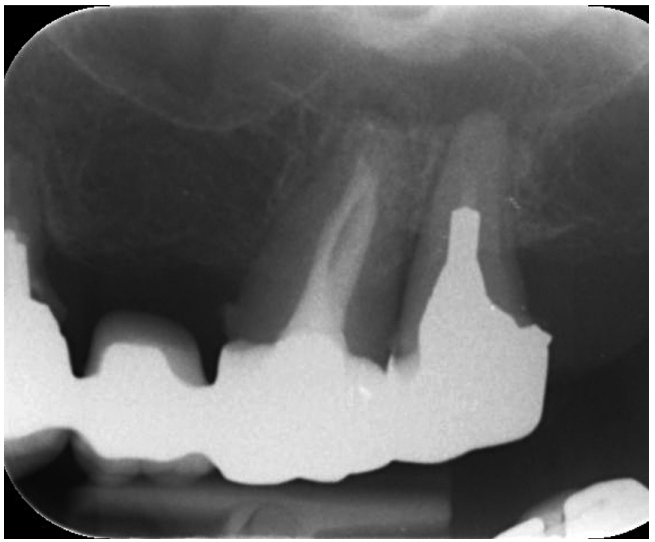

$\triangle$ Fig. 42 : Lésions osseuses péri-apicales 2627.

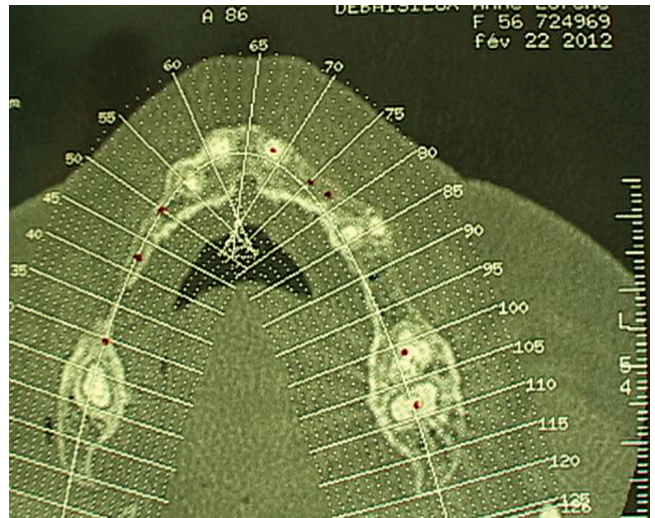

$\triangle$ Fig. 44 : Scanner maxillaire coupe axiale.

Lors du traitement radiculaire, le laser Nd-YAP, grâce à sa fibre de 200 microns de diamètre, prépare le passage de limes en stérilisant les canaux. De plus, associé à l'hypochlorite, les prismes de dentine radiculaire vont exploser sous la pression percutante de l'énergie laser. La désinfection radiculaire est assurée par une pénétration intratubulaire 200 fois plus profonde par rapport aux traitements classiques [4, 5, 6, 7, 16 et 22].

\section{Sinusite iatrogène}

\section{Phase initiale}

Une patiente de 56 ans se présente avec une tuméfaction maxillaire gauche. Elle souffre depuis plusieurs jours. Un bridge de 14 dents repose sur 7 dents. Il est en partie descellé et les piliers sont très mobiles. On note une mobilité axiale sur 25, 26 et 27, accompagnée d'un écoulement purulent gingival. Les radios rétroalvéolaires révèlent un manque d’obturation des canaux (fig. 42 et 43).

Le scanner prescrit en phase initiale (fig. 44) montre un épaississement de la membrane sinusienne considérable ainsi qu'une ostéolyse dans la région apicale et radiculaire molaire (fig. 45, 46 et 47).

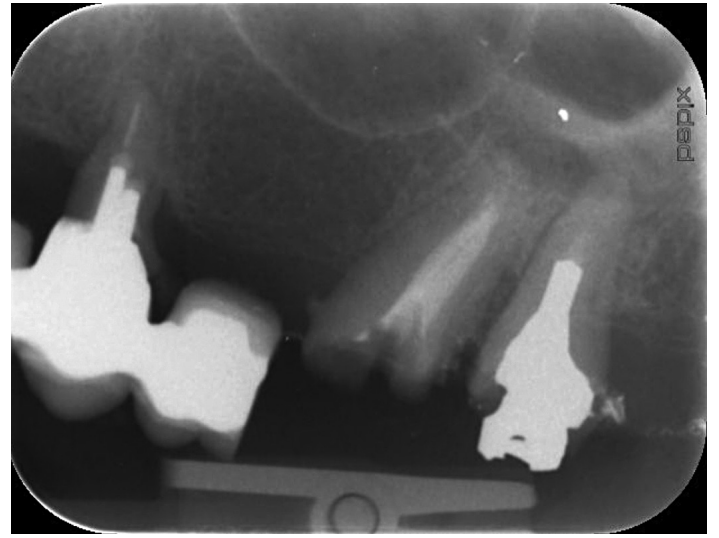

$\triangle$ Fig. 43 : Dépose des couronnes après section du bridge en distal de 25, élimination de la pâte d'obturation canalaire de 26 et dépose de l'amalgame radiculaire en 27.

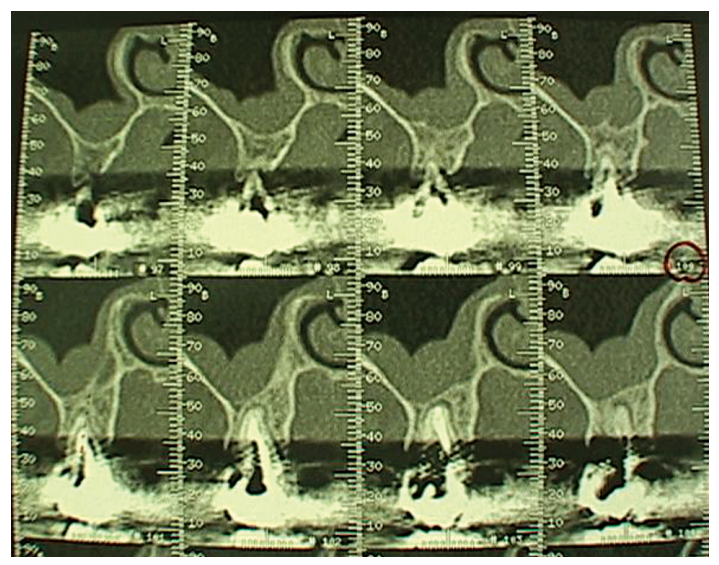

$\triangle$ Fig. 45 : Coupes coronales et mise en évidence de la sinusite d'origine dentaire ; fort épaississement de la membrane sinusienne.

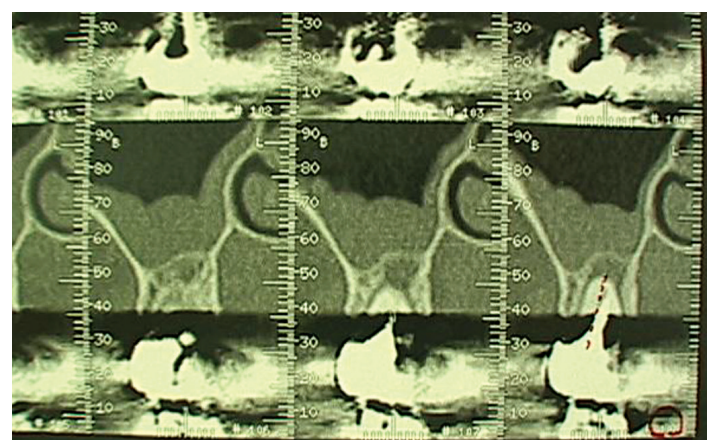

$\triangle$ Fig. 46 : Agrandissement de l'important épaississement de la membrane de Schneider.

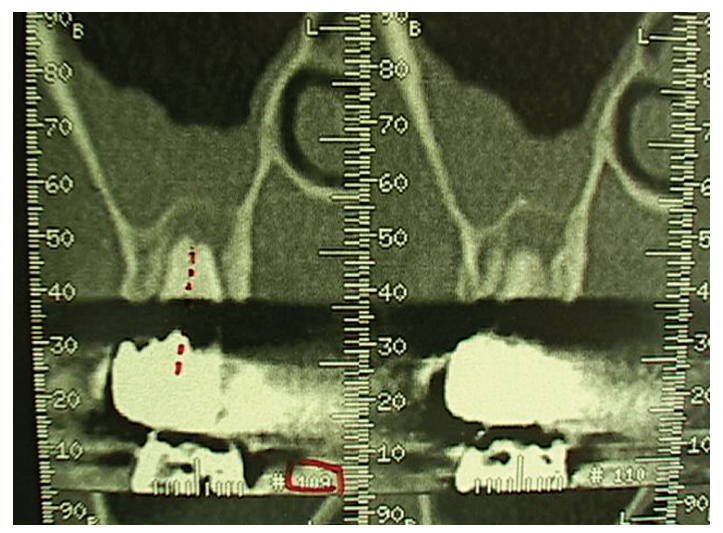

$\triangle$ Fig. 47 : Agrandissement de la lésion en 27. 
Reprise de traitement endodontique

Après section du bridge et dépose des couronnes molaires, lélimination des obstacles radiculaires est rendue très aisée pour la 26 grâce à l'action photothermique du laser qui ramollit pâte et cônes de gutta (fig. 48), mais plus difficile pour la 27 dont le canal palatin a été bouché avec de l'amalgame bourré jusqu'à mi-hauteur radiculaire (fig. 43). En fin de séance, les canaux désobturés, élargis et stérilisés ont été obturés de façon étanche (fig. 49) et les suites ont montré que la sinusite venait bien des anciens travaux iatrogènes. Quatre inlay-cores sont scellés (fig. 50) et un bridge provisoire en résine cuite est réalisé (fig. $\mathbf{5 1}$ ).

\section{Évolution}

Dix-neuf mois plus tard, dans la perspective de poser des implants maxillaires, réalisation d'un scanner (fig. 52) qui objective sur les coupes coronales un assainissement total des sinus (fig. 53 et 54).

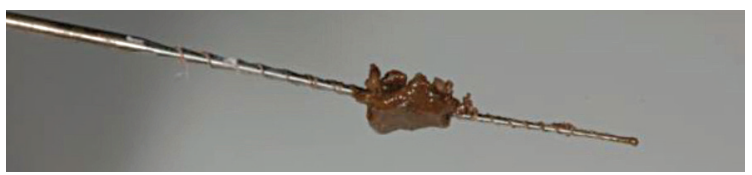

$\triangle$ Fig. 48 : Boue intraradiculaire récupérée lors du traitement canalaire.

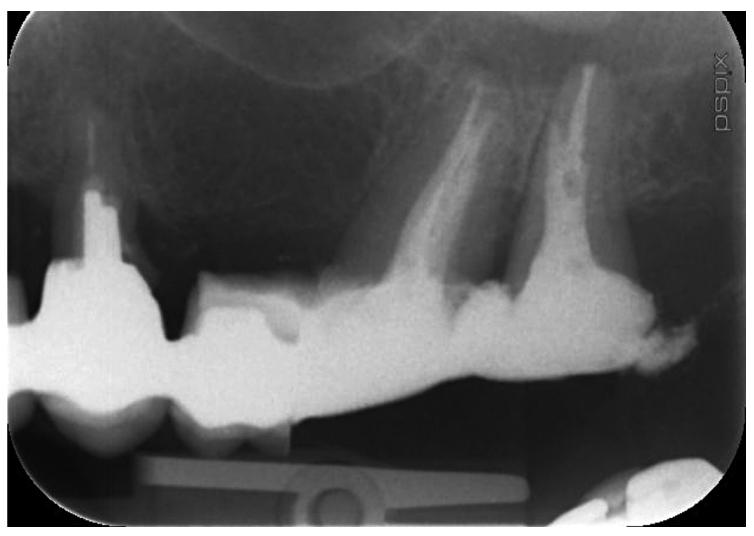

Fig. 49 : Radio après traitement laser des 26 et 27.

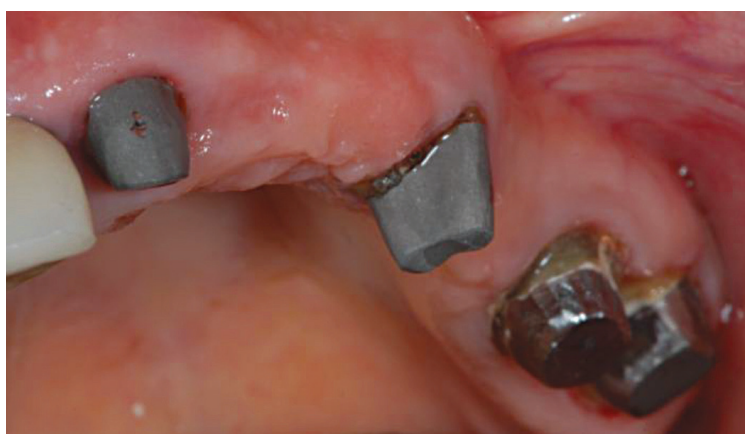

$\triangle$ Fig. 50 : Scellement de 4 inlay-cores après assainissement de $22,24,26$ et 27 .

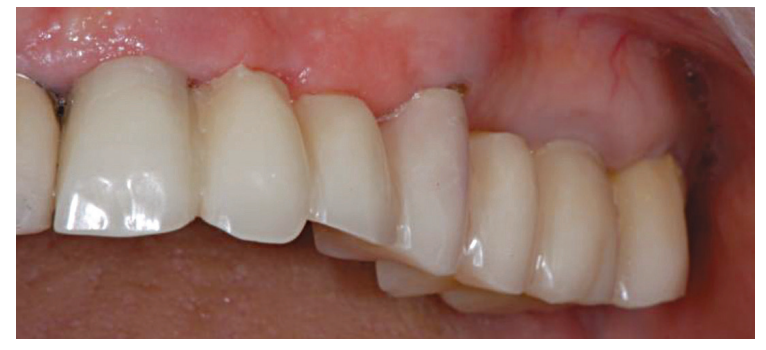

$\triangle$ Fig. 51 : Réalisation d'un bridge provisoire.

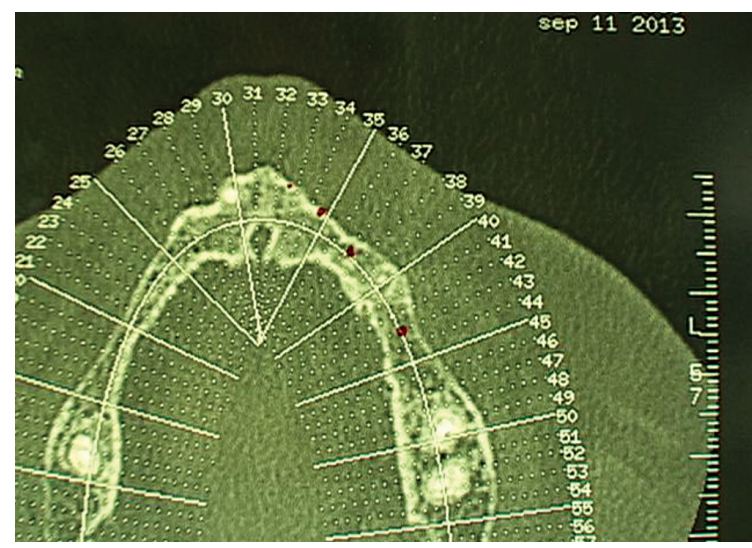

$\triangle$ Fig. 52 : Scanner de contrôle à 19 mois après traitement radiculaire et dentinaire laser associés.

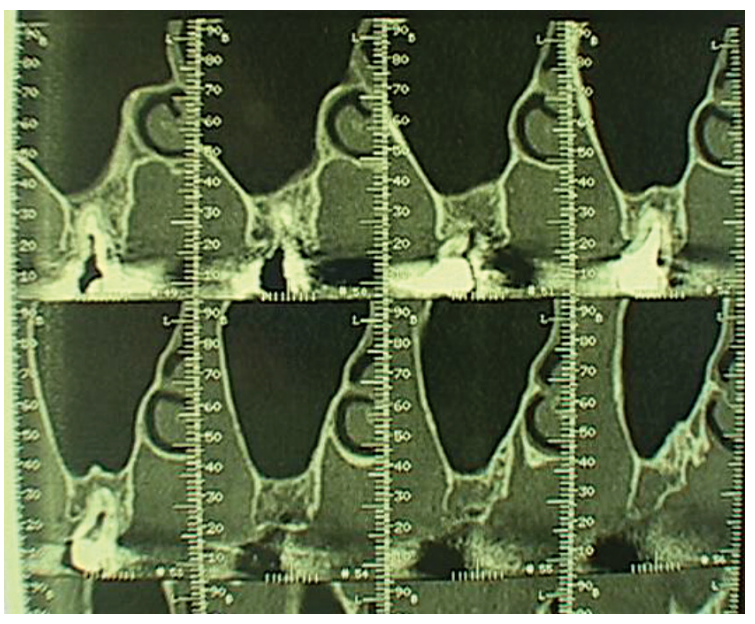

$\triangle$ Fig. 53 : Coupes coronales montrant la disparition de la sinusite en 27.

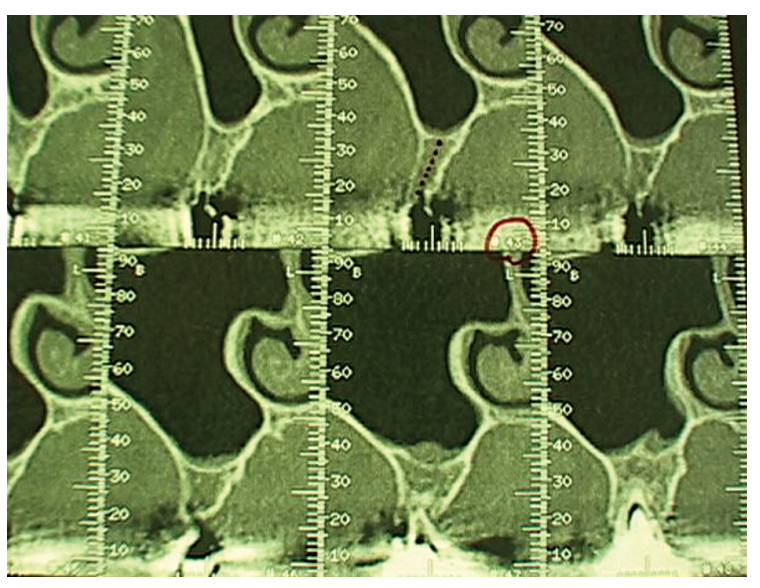

$\triangle$ Fig. 54 : Assainissement en 26. 


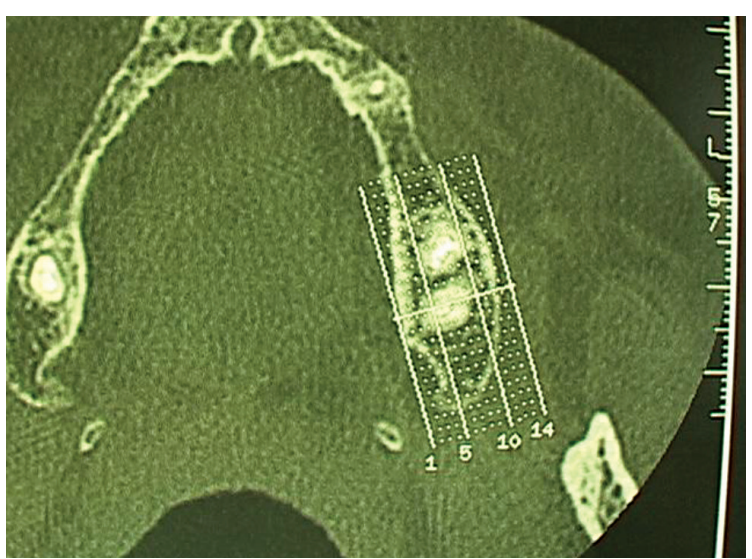

$\triangle$ Fig. 55 : Coupe axiale et sélection de la zone médiale à analyser.

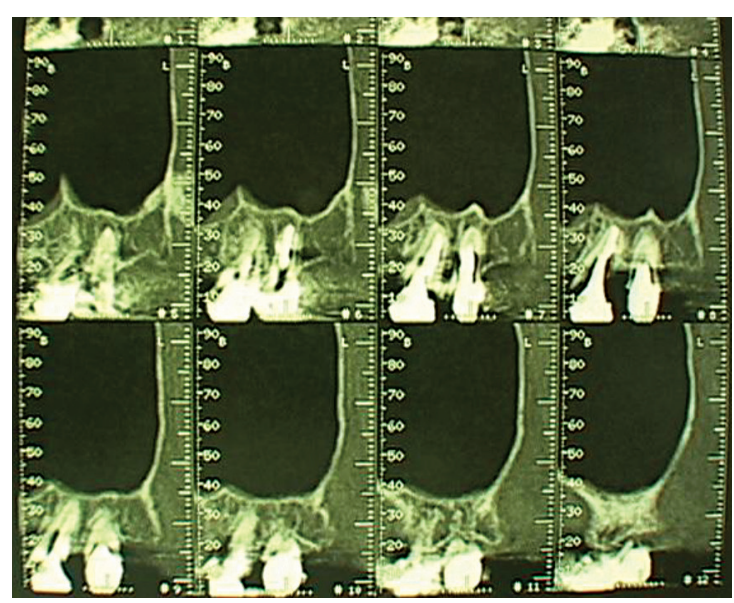

$\triangle$ Fig. 56 : Pneumatisation favorable du sinus gauche.

D’autres coupes sagittales (fig. 55) construites à partir de coupes coronales montrent une pneumatisation favorable du sinus (fig. 56).

\section{Évolution et conclusion}

L'utilisation conjointe de deux lasers pour ce cas, le laser fibré Nd-YAP associé à la vaporisation explosive générée par le laser Er-YAG, en complément d'une technique classique d'alésage radiculaire manuelle et mécanisée, a permis, par une obturation étanche et complète de ces canaux enfin débarrassés de leur contenu pathologique, de résoudre les abcès radiculaires des 26 et 27 et par extension de traiter favorablement les sinus.

Il n'y a eu ni complications postopératoires, ni exploration invasive des sinus, ni chirurgie osseuse ou résections apicales qui auraient engagé le processus de conservation de ces dents.

Laction laser a permis une régénération osseuse sans séquelle ni mutilation. Actuellement, ces dents servent d'ancrage à un bridge de 14 dents, ce qui permet de poser 6 implants dans les zones édentées sans avoir à recourir à une prothèse amovible d'usage qui mettrait en danger l'ostéointégration des implants dans ces zones de crêtes très fines.

\section{Traitement de péri-implantite}

Le biofilm bactérien entraîne une inflammation locale des tissus mous, c'est la « mucosite péri-implantaire », puis intervient la susceptibilité de l'hôte et la persistance de l'agression bactérienne fait progresser la lésion inflammatoire à l'os marginal (J. Lindhe).

En 2010, Ungvari démontre que les surfaces traitées par $\mathrm{H}_{2} \mathrm{O}_{2}$ ont une croissance des cellules épithéliales plus élevées qu'avec la CHX. Les études de Rey montrent une potentialisation des effets en associant $\mathrm{H}_{2} \mathrm{O}_{2} 10$ volumes avec le laser; ce qui permet d'avoir une décontamination très rapide et un «boost» de la cicatrisation.

Lors des péri-implantites, les techniques conventionnelles sont plus longues, plus destructrices et moins efficaces que les techniques laser associées [17 et 38]. Cependant, le laser Er-YAG [40] génère moins d’impact sur le titane, car moins thermique que le YAP qui blesse le titane et crée des plages de fonte de titane et des états modifiés de surface.

En cas de mise en fonction, l'Er-YAG n'endommage pas l'état de surface du titane.

Une application de $\mathrm{H}_{2} \mathrm{O}_{2}$ chauffé à $80^{\circ} \mathrm{C}$ par le laser rétablit la couche d'oxyde de titane d'origine d'après J. Mouhyi [23 et 24].

Le laser Er-YAG décontamine efficacement le PG des surfaces rugueuses, améliore la fixation des ostéoblastes et la prolifération des fibroblastes.

On note un effet bactéricide par la thérapie photodynamique (P. Meisel). Les bactéries Gram+ et les bactéries parodontales pathogènes Gram- sont inactivées et la charge bactérienne est réduite (J.M. Almeida).

Le cas présenté ci-après est le traitement d’une péri-implantite subterminale (fig 57), la reconstruction ad integrum de l'os péri-implantaire et sa stabilité sur 14 ans :

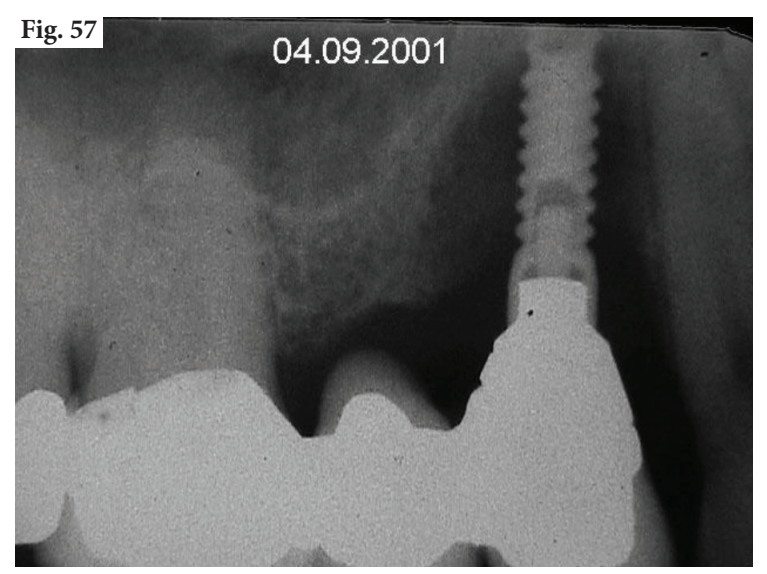

Situation initiale de la péri-implantite sur 14 pilier du bridge implanto-dento-porté posé une douzaine d'années plus tôt. Après avoir soulevé un lambeau mucopériosté et éliminé le tissu de granulation péri- 

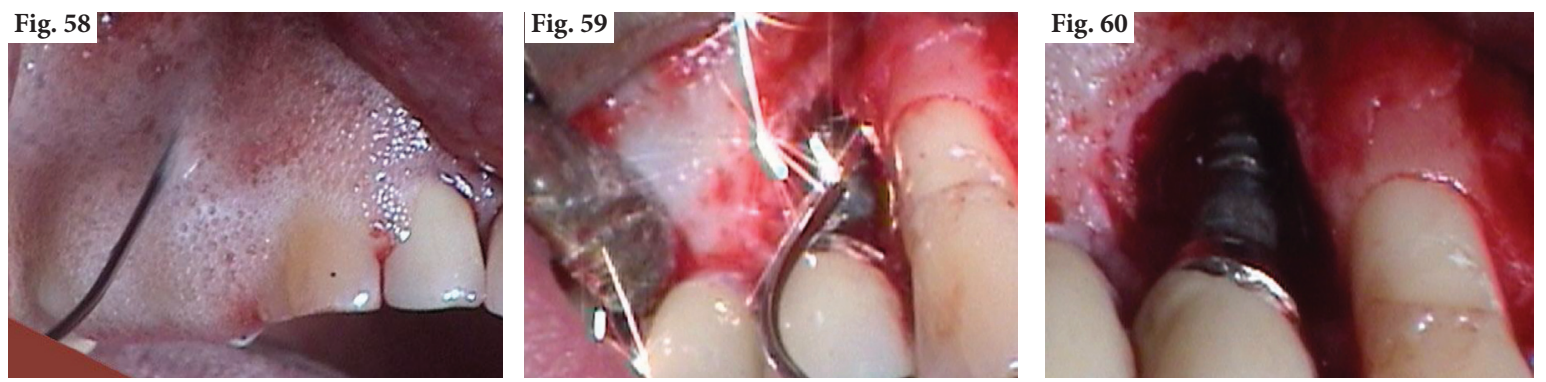
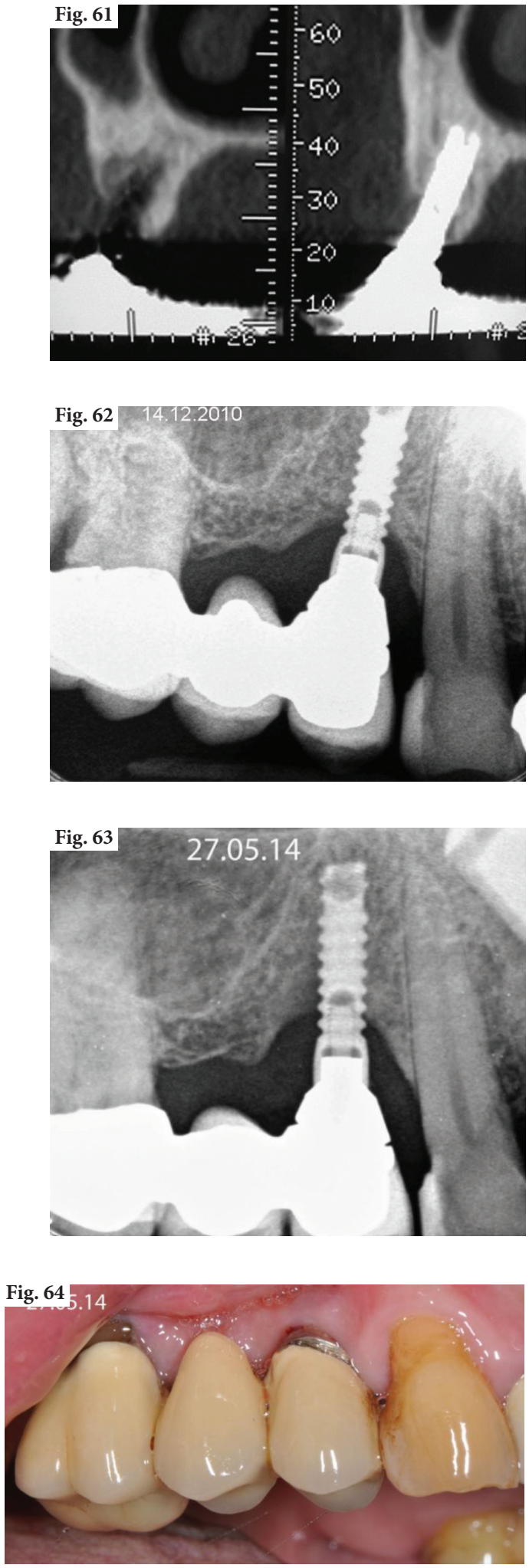

implantaire, les spires de l'implant sont irriguées avec une solution d'eau oxygénée à 10 volumes et la fibre du laser est plongée dans ce bain (fig. 58). Activée, lénergie laser va désagréger le biofilm, anéantir les colonies bactériennes, modifier l'état de surface du titane et biostimuler les tissus osseux et gingivaux [13].

Cette vue (fig. 58) montre le champ opératoire totalement inondé d' $\mathrm{H}_{2} \mathrm{O}_{2} 10$ volumes. La fibre de 300 microns s'active en balayant par rafales la zone à traiter (fig. 59). Quand on tire à sec, le titane subit une surchauffe. Les impacts créent des plages de fusion sur lesquelles se réfléchit le rayonnement laser, ce qui explique la nécessité d'irriguer abondamment les sites à irradier. En fin de séance, les spires exposées sur $12 \mathrm{~mm}$ sont assainies (fig. 60) et une nouvelle action laser à faible fluence permet un saignement contrôlé et maîtrisé qui envahit peu à peu le site comme une sorte de nappe recouvrant les spires de l'implant. Le saignement est transformé en membrane biologique riche en facteurs de croissance, immédiatement recouvert par le lambeau et suturé. Il n'y aura ni comblement osseux, ni pose de membrane.

Une seule intervention a été nécessaire. Ce patient est contrôlé tous les mois pour évaluer la situation radiologique (fig. 62 et 63) et clinique (fig. 64). Deux scanners sont réalisés à 12 ans d'intervalle (fig. 61 et 65).

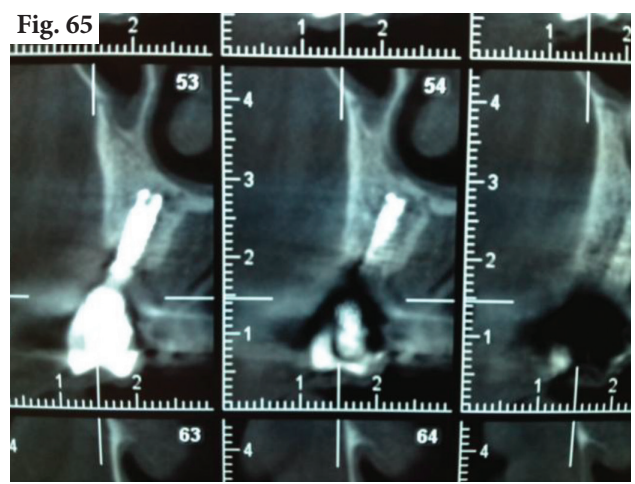

$\triangleleft$ Fig. 61 : Scanner coupe coronale à 9 mois.

$\triangleleft$ Fig. 62 : Reconstruction osseuse stable à 9 ans.

$\checkmark$ Fig. 63 : Contrôle radio à la $14^{\mathrm{e}}$ année.

$\triangleleft$ Fig. 64 : Vue clinique à 13 ans postopératoire.

$\triangle$ Fig. 65 : Analyse scanner à la $14^{\mathrm{e}}$ année qui objective un recouvrement des spires cliniquement absent 13 ans plus tôt. 


\section{CONCLUSION}

Le laser est devenu un auxiliaire thérapeutique incontournable, tant en omnipratique qu'en spécialité, mais ses innombrables qualités demeurent encore méconnues de bon nombre de confrères.

Pendant mes trente premières années d'exercice, je n'ai pas utilisé de laser, pensant même qu'il était superflu, voire inefficace.

Je partageais l'opinion de la majorité de mes confrères qui, encore aujourd'hui, sont très critiques à l'égard d'un instrument qu'ils ne connaissent pas encore.

À l'université, j’ai rencontré quelques enseignants qui restent sceptiques quant à l'utilisation du laser et sont hermétiques à son enseignement.

Depuis treize ans d'utilisation quotidienne des lasers, je constate les bienfaits thérapeutiques obtenus, tant en omnipratique (caries, prothèse, empreintes) qu'en spécialités : parodontologie, endodontie ou chirurgie implantaire.

Laction laser potentialise favorablement le traitement conventionnel [19, 20, 21 et 38] pour optimiser les résultats.

Traitement de carie juxtapulpaire sans altération du parenchyme vasculonerveux [31]. Cicatrisation accélérée sans douleur, ni œdème, après chirurgie. Traitement endodontique plus rapide, plus efficace et plus stable dans le temps. Meilleur collage lors de soins esthétiques.

La réussite d'un traitement demeure cependant conditionnée à l'adéquation entre le cas clinique, la pose d'une bonne indication et le choix du matériel laser adapté ; ce qui implique et nécessite une bonne connaissance par le praticien des modes d'action respectifs de chaque laser, ses principales indications et ses limites thérapeutiques.

Le laser Nd-YAP étant plus pénétrant que l'Erbium-YAG et moins pénétrant que le Nd-YAG ou la diode, sera un excellent outil en omnipratique : cariologie, endodontie, parodontie ou orthodontie.

L'Erbium-YAG trouvera plus d'application en chirurgie reconstructrice et en implantologie, permettant de s'affranchir des greffes et des membranes au pronostic parfois défavorable [35]. Ce laser non absorbé par le titane est un instrument de choix lors des mises en fonction et dans les désinfections des spires implantaires dans les cas de péri-implantites.

Aujourd'hui, parmi l'arsenal thérapeutique mis à notre disposition et au regard de ma pratique quotidienne, seul le laser, en synergie avec les traitements classiques, permet de réaliser la conservation de dents condamnées, mobiles, ou d'implants atteints de péri-implantite avancée au pronostic défavorable. Enfin je formule le vœu qu'un nombre toujours croissant de praticiens fassent le choix d'améliorer la vie de leurs patients en utilisant des techniques laser assistées, fassent connaître leur choix et leur traitement à des confrères de plus en plus nombreux et curieux, car je suis intimement persuadé que recherche, innovations, formations et partage des connaissances sont indissociables et indispensables pour faire avancer la science.

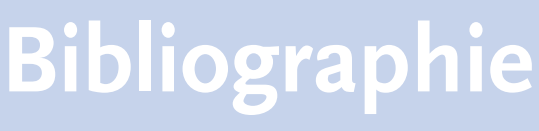

[1] Armengol V, Jean A. Effet du laser Nd-YAP sur l'émail et la dentine. Faculté de chirurgie dentaire de Nantes, 1996.

[2] Atwood DA. Reduction of residuel ridges: a major oral disease entity. J Prosth Dent 1971;26:266-279.

[3] Bert M. La stimulation endostée en Implantologie; études et résultats après 2 ans. Cah Prothèse, 1989;65:23-30.

[4] Blum JY, Abadie JM. Study of the N: Yap Laser. Effects on canal cleanliness. J Endod 1997;23:669-675.

[5] Blum JY, et al. An evaluation of the bactericidal effect of the Nd:Yap Laser. J Endod 1997;23:583-585.
[6] Blum JY, et al. Effects of the N: YAP laser on coronal restorative materials: implications for endodontic retreatment. J Endod 2000;26:588-592.

[7] Caccianiga GL, Papagna R, Baldani A. Analyse de la littérature et évaluation in vivo des effets de l'application du laser dans les soins conservateurs. Dr OS 2002;621-629.

[8] Calas P, Rochd T. Utilisation d'un laser Nd-YAP pour la préparation canalaire : étude préliminaire. Trib Dent 1995;3(20):17-23.

[9] Convissar RA. Principles and Practice of laser dentistry. Mosby Elsevier, 2011.

[10] Crespi R, Covani U, Margarone JE, Andreana S. Periodontal tissue regeneration on beagle dog laser therapy. Laser Surg Med 1997;21:395-402.

[11] Curti M, Rocca JP, Suermondts ABD. SEM Study of root canals lased with argon. In: Rechmann P, Hennig D, eds. Lasers in dentistry. VII. SPIE 2001;4249:8-14
[12] Darbar R. Hopper C, Speight PM, Newmann HN. Combined treatment approach to gingival overgrowth due to drug therapy. J Clin Periodontol 1996;23:941-4.

[13] Deppe H., Horch HH. Laser applications in oral surgery and implant dentistry. Lasers Med Sci. 2007 Nov;22(4):217-21.

[14] Farge P, Nahas P, Bonin P. In vitro study of a ND-YAP laser in endodontics retreatment. J Endod 1998;24:359-363.

[15] Kiernicka M, Owczarek B, Galkowska E, Wysokinska-Miszczuk J. Comparison of the effectiveness of the conservative treatment of the periodontal pockets with or without the use of laser biostimulation. Ann Univ Mariae Curie Sklodowska [Med] 2004;59(1):488-94

[16] Mader CL, Baumgartner JC, Peters DD. Scanning electron microscopic investigation of the smeared layer on root canal walls. J Endod 1984;10:477-83. 


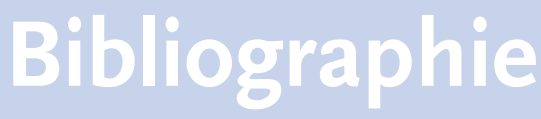

[17] Missika P, Stroumza JM. Traitement de péri-implantite avec un laser Nd :YAP. ROS 2003;32:215-229.

[18] Missika P, Rey G, Stroumza JM. Collection $J P I O:$ Les lasers et la chirurgie dentaire; 2010. Chapitre 3 : place réelle du laser en omnipratique quotidienne. Chapitre 6 : Intérêt du laser dans le traitement de la péri-implantite.

[19] Missika P, Stroumza JM. 30 questions en implantologie. ID 2011.

[20] Missika P, Stroumza JM. 25 cas complexes en implantologie ? ID 2012.

[21] Missika P, Stroumza JM. 22 cas d'école en implantologie. ID 2013.

[22] Moshonov J. et al. Cleaning of the root canal using Nd:YAP laser and its effect on the mineral content of the dentin. J Clin Laser Med Surg 2003;21:279-282.

[23] Mouhyi J. et al. Temperature increases during surface decontamination of titanium implants using $\mathrm{CO}_{2}$ laser. Clin Oral Implants Res 1999;10(1):54-61.
[24] Persson LG, Mouhyi J, Berglundh T, Sennerby L, Lindhe J. Carbon dioxide laser hydrogen peroxide conditioning in the treatment of periimplantitis; an experimental study in the dog. Clin Implant Dent Relat Res 2004;6(4):230-8.

[25] Pietrokowski J, Massler M. Alveolar ridge resorption following tooth extraction. J Prosthet Dent 1967;17(1):21-27.

[26] Rey G, Missika P. Traitements parodontaux et lasers en omnipratique dentaire. Masson, 2010.

[27] Rey G. Efficacité des lasers en parodontologie. La lettre de la stomatologie 2009;43:4-21.

[28] Ricardo A. et al. In vivo study of the Nd:YAP laser in persistent periapical lesion. Photomed Laser Surg 2005;23(6):582-585.

[29] Sauvetre E, Rastegar Babak, El Yazami H. Évaluation clinique du laser YAP en Parodontologie. ID 2004;(16):1077-1082.

[30] Schwarz F, Becker J. Peri-implant Infection. Quintessence publishing, 2007.

[31] Seux D, Romeas A, Antoine B, Magloire $\mathrm{H}$, Bleicher $\mathrm{F}$. In vitro study of a neodymium: yttrium aluminium perovskite laser on human non exposed pulp after cavity preparation. Clin Oral Invest 2004;8:145-150.
[32] Stroumza JM. Cas complexe. Traitement simple. AOS 2006;233:17-24.

[33] Stroumza JM. Option implantaire dans un cas limite. ID 2006;88(3):74-79.

[34] Stroumza JM. Traitements implantaires simplification d'un cas complexe. AOS 2003;223.

[35] Stroumza JM. Prévention et gestion des complications de greffes osseuses. AOS 2006;235:287-294.

[36] Stroumza JM. Les 10 points clés en implantologie. Coll. Implantologie sans greffe. Européenne Édition nov 2009.

[37] Stroumza JM. Laser Nd YAP et réimplantation. ID 2010;5.

[38] Stroumza JM. Biostimulation Laser et implantologie. Alpha Oméga News 2010;132.

[39] Stroumza JM. Cas clinique en Implantologie ou comment transformer l'échec en succès. AOS mars 2012.

[40] Sulc J, et al. Comparison of diode-sidepumped Nd:YAG and Nd:YAP laser - Solid State Lasers XIV: Technology and Devices. Edited by Hoffman, Hanna J, Shori, Ramesh K. Proceedings of the SPIE 2005;5707:325-334. 